Paediatr Paedolog 2015 · [Suppl 1] 50:3-3 DOI 10.1007/s00608-015-0233-z

(c) Springer-Verlag Wien 2015

\title{
W. Sperl
}

Zentrum für Kinder- und Jugendmedizin, Universitätsklinik für Kinder- und Jugendheilkunde, Paracelsus Medizinische Privatuniversität (PMU), Salzburger Landeskliniken (SALK), Salzburg

\section{Die Leitlinien zur Ernährung in der pädiatrischen Palliativmedizin}

sen, aber auch ein spezielles Wissen notwendig. Alle Autoren haben aus ihren Bereichen ihre reichen Erfahrungen und ihr Spezialwissen über Palliativpatienten beigetragen. Dies zeichnet diese Leitlinie aus, wie auch die Zielrichtung und der rote Faden: die „Wertschätzung und Empathie für die Patienten“, der „tiefe Wunsch, eine Verbesserung auch für diese Patientengruppen zu erzielen“ und das Bemühen, hier mehr zu bewegen.

Die pädiatrische Palliativmedizin ist in Österreich ein Entwicklungsgebiet. Es gibt momentan wertvolle Bestrebungen, Verbesserungen sowohl im ambulanten als auch im stationären Bereich für palliativmedizinische Patienten umzusetzen. Wesentlich ist auch, dass in den Österreichischen Strukturplan Gesundheit (ÖSG) die Palliativmedizin für Kinder- und Jugendliche mit aufgenommen wird. Die Leitlinien sind ein wichtiger Beitrag, der direkt den Patienten zugutekommen wird. Die Ernährung ist auch ein besonderes „Familienthema“. Diese Leitlinien können zur besseren Versorgung und zur Unterstützung der Familien und der multiprofessionellen Betreuungsteams beitragen. Der Untertitel „Interdisziplinäres fachübergreifendes Projekt" sagt schon aus, dass hier eine Dynamik und eine Kontinuität mit entsprechenden Updates notwendig sein werden.

Ich wünsche dem Vorhaben nachhaltiges Gelingen.

\section{Mit freundlichen Grüßen}

Prim. Univ.-Prof. Dr. Wolfgang Sperl Präsident der Österreichischen Gesellschaft für Kinder- und Jugendheilkunde (ÖGKJ)

\section{Korrespondenzadresse}

Prim. Univ. Prof. Dr. Wolfgang Sperl

Zentrum für Kinder- und Jugendmedizin Universitätsklinik für Kinder- und Jugendheilkunde

Paracelsus Medizinische Privatuniversität (PMU)

Salzburger Landeskliniken (SALK)

Müllner Hauptstraße 48

5020 Salzburg

Salzburg

Tel.: +43662 44822600

Fax: +43 66244822604

E-Mail:w.sper@@salk.at

Interessenkonflikt. W. Sperl gibt an, dass kein Interessenkonflikt besteht. 